\title{
Image of the month: Brachiocephalic arterial occlusive limb-shaking transient ischaemic attacks
}

\author{
Authors: Ahmed Abbas, ${ }^{\mathrm{A}}$ Kurdow Nader, ${ }^{\mathrm{B}}$ Alok Tiwari, ${ }^{\mathrm{C}}$ Arunagiri Viruthagiri ${ }^{\mathrm{D}}$ and Edward Littleton ${ }^{\mathrm{E}}$
}

\begin{abstract}
A 75-year-old man with multiple cardiovascular risk factors presented with a 2-year history of increasingly frequent shaking episodes simultaneously affecting left upper and left lower limbs, lasting up to 30 seconds. There was no impairment of consciousness, speech or any other neurological disturbance in relation to these attacks but they were often precipitated by a change in posture. Limb-shaking transient ischaemic attacks (LS-TIA) were suspected.
\end{abstract}

Cervical doppler ultrasonography revealed significantly dampened flow in right common and internal carotid arteries. Computed tomography (CT) angiography showed dense calcification of the brachiocephalic (or innominate) artery, but no significant stenosis of the common or internal carotid arteries. Further assessment with invasive catheter angiography was undertaken, during which contrast injection into the aorta identified a proximally occluded brachiocephalic artery (Fig 1a). In addition, later downward filling of the distalmost brachiocephalic artery, and subsequently right subclavian artery, was illustrated by retrograde flow down the right cervical (common carotid and vertebral) arteries (Fig 1b). This retrograde flow, previously dubbed the 'innominate steal phenomenon,', effectively reflected the right upper limb 'stealing' its arterial supply from the right cerebral hemisphere.

An endovascular approach to revascularisation was deemed unsuitable due to heavy brachiocephalic calcification. Therefore, the patient underwent an extra-anatomical cervical left-toright common carotid bypass procedure with a prosthetic graft. Postoperative recovery was uneventful and the procedure was successful in completely resolving his LS-TIA. Postoperative CT brain perfusion imaging also showed improved right hemispheric cerebral blood flow (Fig 2).

Almost all reports of LS-TIA relate to occlusive disease of the extracranial or intracranial carotid arterial system, with reduced forward arterial flow to the hemispheres. ${ }^{2,3}$ This case arising secondary to brachiocephalic arterial occlusion, with additional backward hemispheric arterial steal, is unusual and only one

Authors: AST6 clinical neurophysiology, Queen Elizabeth Hospital Birmingham, Birmingham, UK; ${ }^{\mathrm{B}}$ Consultant neuroradiologist, Queen Elizabeth Hospital Birmingham, Birmingham, UK; ' ${ }^{C}$ consultant vascular surgeon, Queen Elizabeth Hospital Birmingham, Birmingham, UK; ${ }^{\text {D }}$ Senior vascular fellow, Queen Elizabeth Hospital Birmingham, Birmingham, UK; ${ }^{E}$ consultant neurologist, Queen Elizabeth Hospital Birmingham, Birmingham, UK

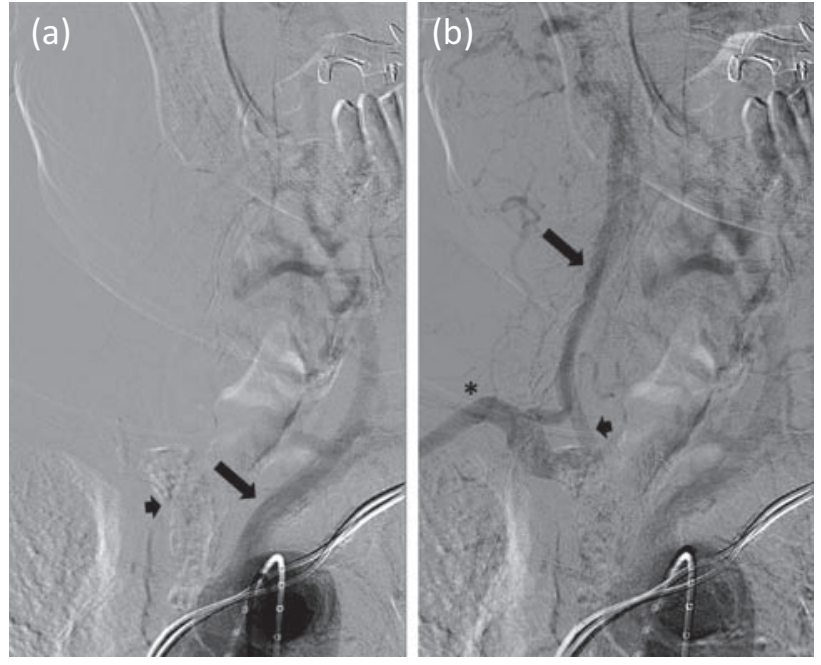

Fig 1. Catheter angiogram. (a) No filling of the calcified brachiocephalic artery (short arrow) despite filling of left common carotid artery (long arrow), followed shortly by (b) retrograde flow down the right vertebral artery (long arrow) and right common carotid artery (short arrow) to fill the distalmost brachiocephalic and ultimately right subclavian artery (asterisk).

other case is recently reported in the literature. ${ }^{4}$ Awareness of extra-carotid arterial stenoses as potential causes of LS-TIA is important in accurately identifying the causally occluded arterial segment, and consequently in selecting the most appropriate
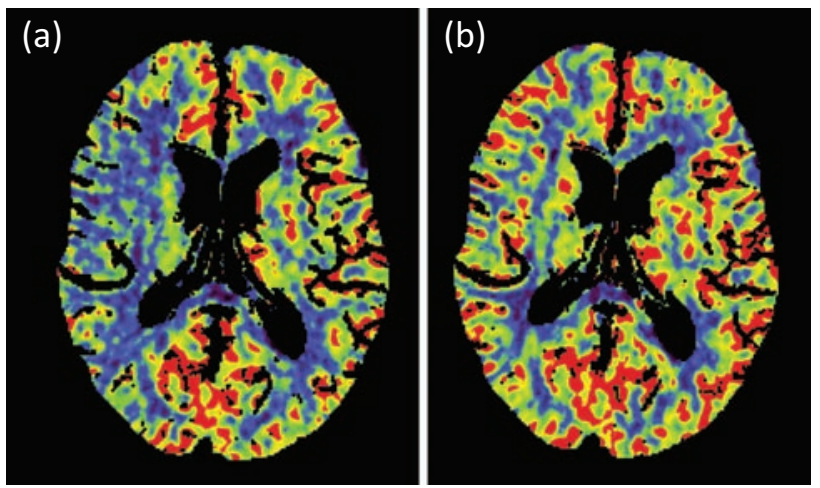

Fig 2. Computed tomography brain perfusion imaging. (a) Preoperative impaired right cerebral hemispheric blood flow (blue). (b) Postoperative improvement. 
surgical revascularisation procedure. In cases such as ours, wider diagnostic studies encompassing arterial vasculature proximal to the carotid arteries may be more sensitive in this regard compared to focal carotid arterial examinations. ${ }^{4}$

\section{Consent}

Consent was obtained from the patient to publish the clinical details and images in this article.

\section{References}

1 Uzun M, Bağcier S, Akkan K et al. Innominate steal phenomenon: color and spectral Doppler sonographic findings. J Ultrasound Med 2008;27:1537-8.
2 Baquis GD, Pessin MS, Scott RM. Limb shaking - a carotid TIA. Stroke 1985;16:444-8

3 Salah Uddin AB. Limb shaking transient ischemic attack - an unusual presentation of carotid occlusive disease. A case report and review of the literature. Parkinsonism Relat Disord 2004;10:451-3.

4 Goldstein ED, Cannistraro R, English S et al. Brachiocephalic arterial occlusive disease presenting as limb-shaking transient ischemic attacks. J Stroke Cerebrovasc Dis 2018;27:e34-5.

Address for correspondence: Dr Ahmed Abbas, Department of Clinical Neurophysiology, Queen Elizabeth Hospital Birmingham, Birmingham B15 2GW, UK.

Email:ahmed.abbas4@nhs.net 